\title{
Effects of Dietary Phosphate on Ectopic Calcification and Muscle Function in mdx Mice
}

\author{
Eiji Wada, Namiko Kikkawa, Mizuko Yoshida, \\ Munehiro Date, Tetsuo Higashi and Ryoichi Matsuda \\ The University of Tokyo, \\ Japan
}

\section{Introduction}

Calcium deposits in extra-skeletal tissues are highly correlated with lifestyle diseases. The mechanisms and clinical effects of such deposition have been widely studied due to increase mortality rate. Vascular calcification is a major complication in a number of diseases, including chronic kidney disease (CKD) and diabetes (Giachelli, 2009). The number of regulation mechanisms affecting calcium precipitation in soft tissues remains underestimated, as many regulators are considered to be involved in this complex process (Hu et al., 2010; Kendrick et al., 2011). Elevated serum phosphate levels which leads hyperphosphatemia is one of the prevalent factors of vascular calcification in CKD (El-Abbadi et al., 2009). The kidneys play a central role in the regulation of phosphate homeostasis. In individuals with normal renal function, serum phosphate levels are strictly controlled through dietary intake, intestinal absorption, renal excretion, and bone metabolism. When the kidneys are either mechanically or functionally impaired, phosphate metabolism is imbalanced. Abnormalities of phosphate metabolism related to kidney malfunction may play a central role in the deposition of calcium and phosphate in extra-skeletal tissues. Ectopic calcification in skeletal muscle has been reported to occur in three Duchenne muscular dystrophy (DMD) animal models; mdx mice (Coulton et al., 1987; Kikkawa et al., 2009), dystrophic puppies (Nguyen et al., 2002), and hypertrophic muscular dystrophy cats (Gaschen et al., 1992). In this chapter, we review the mechanisms of ectopic calcification in $\mathrm{mdx}$ mice and report a new finding of effects of dietary phosphate intake on calcium deposits and muscle function in $\mathrm{mdx}$ mice.

\section{Ectopic calcification in animal models of muscular dystrophy}

The mdx mouse, dystrophic canine, and hypertrophic muscular dystrophy feline develop progressive muscle lesions and calcium deposits in skeletal muscle during muscle regeneration. The pathological features of dystrophic golden retriever puppies are particularly severe and are similar to those of DMD boys, who are characterized by progressive muscle necrosis that leads to early death. Nguyen et al. (2002) detected early ectopic calcification in muscles from 4-day-old and 2-month-old puppies. Thus calcium deposition in skeletal muscle appears to be an early event associated with muscle degeneration.

In mdx mice, the observed muscle pathology is relatively mild compared with DMD patients but calcifying lesions are commonly seen in the lower limbs and diaphragm of mice from 
approximately five weeks of age. Recently, ectopic calcification (Fig. 1) has been reported to be a characteristic feature of muscular pathology (Korff et al., 2006; Verma et al., 2010). For example, Korff et al. (2006) found that myocardial calcification commonly occurs in mice following necrosis induced by mechanical stresses and proposed that calcification in the heart is dependent upon genetic background. Verma et al. (2010) suggested that the absence of ectopic calcification in the diaphragm serves as a marker of amelioration of mdx pathology. In addition, one of the prednisone-induced side effects in a canine model of DMD is skeletal muscle calcification (Liu et al., 2004). However, a palliative glucocorticoid therapy using prednisone is a feasible and effective treatment approach for DMD despite of the serious potential side effects (Wong et al., 2002; Khan, 1993). Studies in these animals have revealed that the percentages of calcified myofibers in necrotic lesions increase dose dependently. It is speculated that calcium deposits in skeletal muscle are occurred as results of abnormal calcium and phosphate homeostasis and delayed muscle degeneration and regeneration cycle.
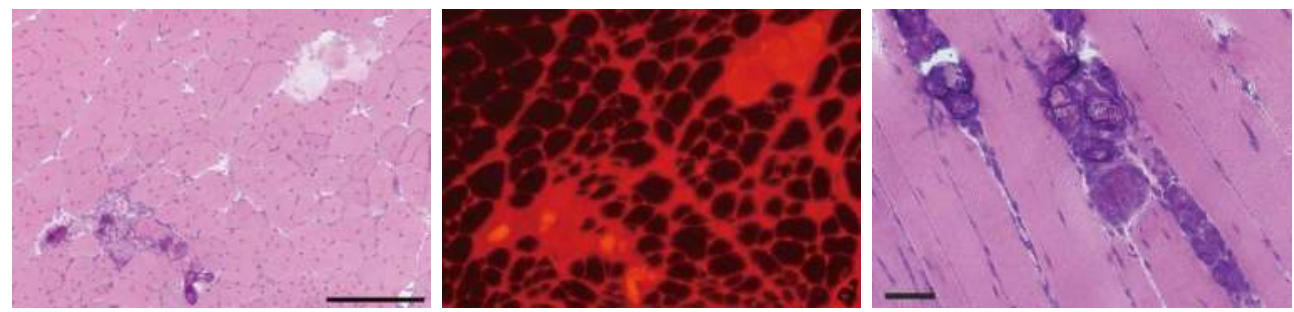

Fig. 1. Ectopic calcification in mdx mice (90 days old). Transverse (left and center) and longitudinal (right) sections, stained with H\&E (left and right) and Evans blue (center). The bar represents $100 \mu \mathrm{m}$.

\section{Identification of calcium deposits in $\mathrm{mdx}$ mice skeletal muscle}

Our group is actively studying ectopic calcification in mdx mice skeletal muscle (Kikkawa et al., 2009). We performed experiments with 90-day-old $\mathrm{mdx}$ and control mice (C57BL/10: B10) fed a commercial standard chow (CE-2; Clea Japan, Tokyo, Japan) and water ad libitum. Following sacrificed of the mice, high-resolution X-ray micro-computed tomography (CT) imaging of the hind limbs of mdx and B10 mice using a SkyScan-1074 scanner (SkyScan, Kontich, Belgium) revealed that all mdx mice had muscle calcification in the hind limb, whereas no calcium precipitation was observed in the control mice (Fig. 2).
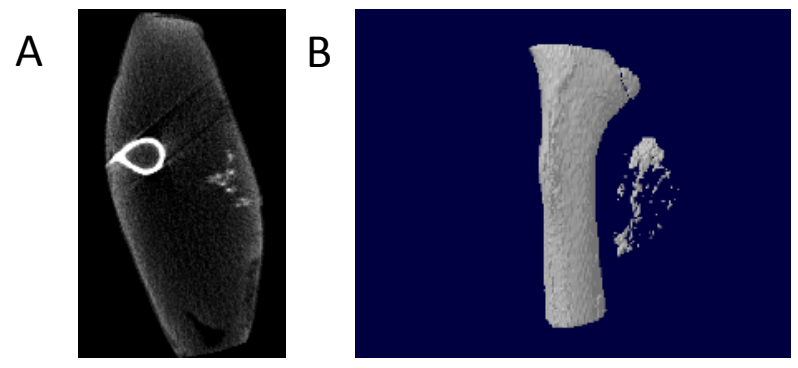

Fig. 2. Images of the hind limb of a two-month-old mdx mouse. X-ray-absorbing materials are shown as gray shadows and the femeur can be seen in the center of the $X$-ray image. (A) CT image. (B) Reconstructed 3D image. (Kikkawa et al., 2009) 
The main composition of calcium deposits in the skeletal muscle was identified using an back-scattered electron imaging and energy-dispersive X-ray spectrometry (EDS) analysis by S-4500 SEM (Hitachi, Tokyo, Japan). In a cross-section of the muscle from an mdx mouse, spotty and bright crystals were observed. The EDS spectra obtained from the crystals indicated the presence of both calcium and phosphorus (Fig. 2A-B). To determine whether the composition of the deposits consisted of a calcium phosphate phase, muscle samples were analyzed using a JEM-2010 TEM (JEOL, Tokyo, Japan) equipped with an EDS detector. The electron diffraction pattern from an obtained TEM image of the specimen nearly was an identical match with a simulated diffraction pattern of hydroxyapatite (Ca5(PO4)3OH; HA) (Fig. 3C). Based on these results, we concluded that the calcification of mdx skeletal muscles is due to the precipitation of hydroxyapatite.

A

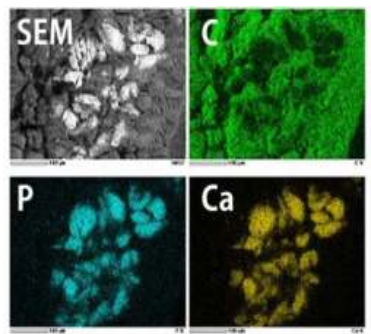

EDS $^{*}$
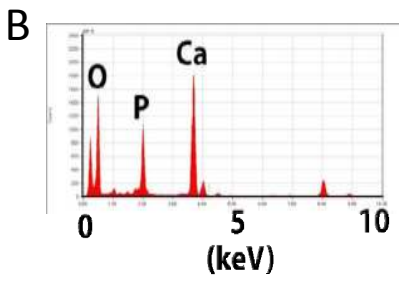

C

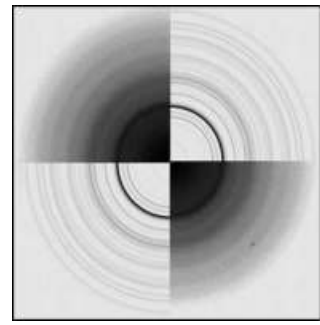

Fig. 3. SEM and TEM analyses of ectopic calcification in mdx mice skeletal muscle. (A) Electron probe microanalysis identified the particles as calcium phosphate. (B) Energy dispersive X-ray spectroscopy. (C) Identical match of X-ray diffraction of the particles and HA. (Kikkawa et al., 2009).

\section{Serum biochemistry of $\mathrm{mdx}$ and B10 mice fed a commercial diet}

As we determined that ectopic calcification is composed of HA, the main component of bones, we suspected that $\mathrm{mdx}$ mice have a metabolic disorder of calcium (Ca) and phosphate $(\mathrm{Pi})$ homeostasis. To examine the levels of $\mathrm{Ca}$ and $\mathrm{Pi}$ in blood, serum samples were collected from two-month-old $\mathrm{mdx}$ and B10 mice fed a commercial diet (CE-2 containing $1.0 \mathrm{~g} / 100 \mathrm{~g} \mathrm{Pi}$ and $1.0 \mathrm{~g} / 100 \mathrm{~g} \mathrm{Ca}$ ) and water ad libitum. The two minerals were measured using an automated clinical chemistry analyzer Fuji Dri-chem 4000 (Fujifilm, Tokyo, Japan). Comparison of the serum mineral components of $\mathrm{mdx}$ and B10 mice revealed that $\mathrm{mdx}$ mice had significantly higher serum Pi levels $(1.41$ fold; $\mathrm{P}<0.05)$ than the control mice, whereas no significant differences in serum Ca levels were detected. These results are supported by a previous study in mdx and B10 mice by Brazeau et al. (1992).

The concentrations of serum fibroblast growth factor-23 (FGF-23), which is an important regulator of phosphorus, were also measured using an FGF-23 ELISA kit (Kainos Laboratories, Tokyo, Japan). The serum level of FGF-23 of $\mathrm{mdx}$ mice was significantly higher $(1.5$ fold; $\mathrm{P}<0.05)$ than that of B10 mice.

Nearly all of the identified functions of FGF-23 are activated or operate through Klotho, a single transmembrane protein of the $\beta$-glycosidase family that is expressed in the distal kidney tubules and parathyroid gland (Kuro-o, 2010). Both FGF-23 and Klotho have emerged as responsible factors for mediating phosphate homeostasis. It has been reported 
that soft tissue calcification and hyperphosphatemia are observed in mice lacking either FGF-23 (Razzaque et al., 2006) or Klotho (Kuro-o et al., 1997). Klotho mutant mice also exhibit multiple age-associated disorders, such as arteriosclerosis, osteoporosis, short-life span, and ectopic calcification. However, as these phenotypes are rescued by the restriction of dietary phosphorus alone in male Klotho mice (Morishita et al., 2010) we predicted that the amount of dietary Pi intake influences the precipitation of calcium in mdx mice, and that the restriction of dietary Pi may improve $\mathrm{mdx}$ muscle pathology and function.

\section{Influence of phosphate diet}

Based on our findings that mdx mice have calcium deposits composed of HA and exhibit higher serum phosphate levels, we speculated that dietary phosphate intake might modulate ectopic calcification in mdx mice. To test this speculation, mdx mice and B10 mice were divided into three diet groups $(n=30)$ from weaning (20 days old) that were fed diets with Pi contents of $2.0 \mathrm{~g} / 100 \mathrm{~g}$ (high-Pi diet), $1.0 \mathrm{~g} / 100 \mathrm{~g}$ (mid-Pi diet), and 0.7 g/100 g (low$\mathrm{Pi}$ diet) manufactured by Oriental Yeast Company (Tokyo, Japan). Other ingredients, including calcium $(1.2 \mathrm{~g} / 100 \mathrm{~g})$ in the diets were present in the same amounts among the groups. The experimental diets were based on the CE-2 and mid-Pi diet was a same composition with CE-2 diet which was fed to pregnant and nursing mice of both genotypes. All mice were housed in cages with pulp bedding (Palmas- $\mu$; Material Research Center, Tokyo, Japan) in a controlled room with a 12-h light/dark cycle and a temperature of $25^{\circ} \mathrm{C}$. The experimental chows and water were available ad libitum. Mice were either sacrificed with an overdose of diethylether at age 30,60, or 90 days or used for measurements of muscular function at age 60 days. Twenty-four hours before euthanasia, mice were received an intraperitoneal injection of Evans blue dye (EBD, $100 \mathrm{mg} / \mathrm{kg}$ ) which incorporates into regenerating myofibers with permeable membranes (Matsuda et al., 1995). All procedures were performed in accordance with the ethical guidelines of the University of Tokyo.

\subsection{Changes in ectopic calcification in skeletal muscle}

Changes in ectopic calcification in mdx mice skeletal muscle induced by dietary phosphate content were observed using a modified whole body double-staining method involving alizarin red $\mathrm{S}$ and alcian blue, which stain bones and cartilage respectively (Dingerkus et al., 1977; McLeod, 1980; Webb et al., 1994). Briefly, 90-day-old mice were sacrificed and fixed in $95 \%$ ethanol $(\mathrm{EtOH})$ for 7 days after the skin and organs were removed. The EtOH was then replaced in acetone and the samples were further incubated for 3 4 days. After partial drying, samples were stained in a mixed solution of $0.3 \%$ alcian blue 8GX (Fluka, Germany) in $70 \% \mathrm{EtOH}, 0.1 \%$ alizarin red S (WAKO, Osaka, Japan) in $95 \% \mathrm{EtOH}$, and $2.0 \%$ potassium hydrogen phthalate in $70 \% \mathrm{EtOH}$ for 3 days. Each stained mouse was washed in distilled water and placed in $0.75 \%$ potassium hydroxide $(\mathrm{KOH})$ in MilliQ water for 2 days to initiated maceration and clearing. Clearing was continued by adding increasing concentration of glycerol $(20 \%, 50 \%, 70 \%$ and $100 \%)$ in $0.75 \% \mathrm{KOH}$ to obtain a completely cleared specimen (Fig. 4A). Calcified regions were stained reddish violet, similar to appearance of stained bones.

Imaging of the stained and cleared samples showed that no bone-like red staining was present in the skeletal muscles of B10 mice fed any of the three phosphate diets (Fig. 4A-a). However, in mid-Pi fed mdx mice, striped and spotty red stained areas, particularly in the 
back, gluteus, and lower limbs muscles, were detected (Fig. 4A-b), while excessive calcification was clearly observed in the samples from high-Pi fed mdx mice (Fig. 4A-c, Fig. 4B). The staining revealed severe calcification, particularly in the diaphragm, back, gluteus, and lower limbs muscles, where severely degenerated muscle fibers were visible macroscopically by EBD staining (Fig. 4C). In contrast, bone-like red staining was rarely seen in the whole bodies of the low-Pi fed mdx mice (Fig. 4A-d, Fig. 4B).

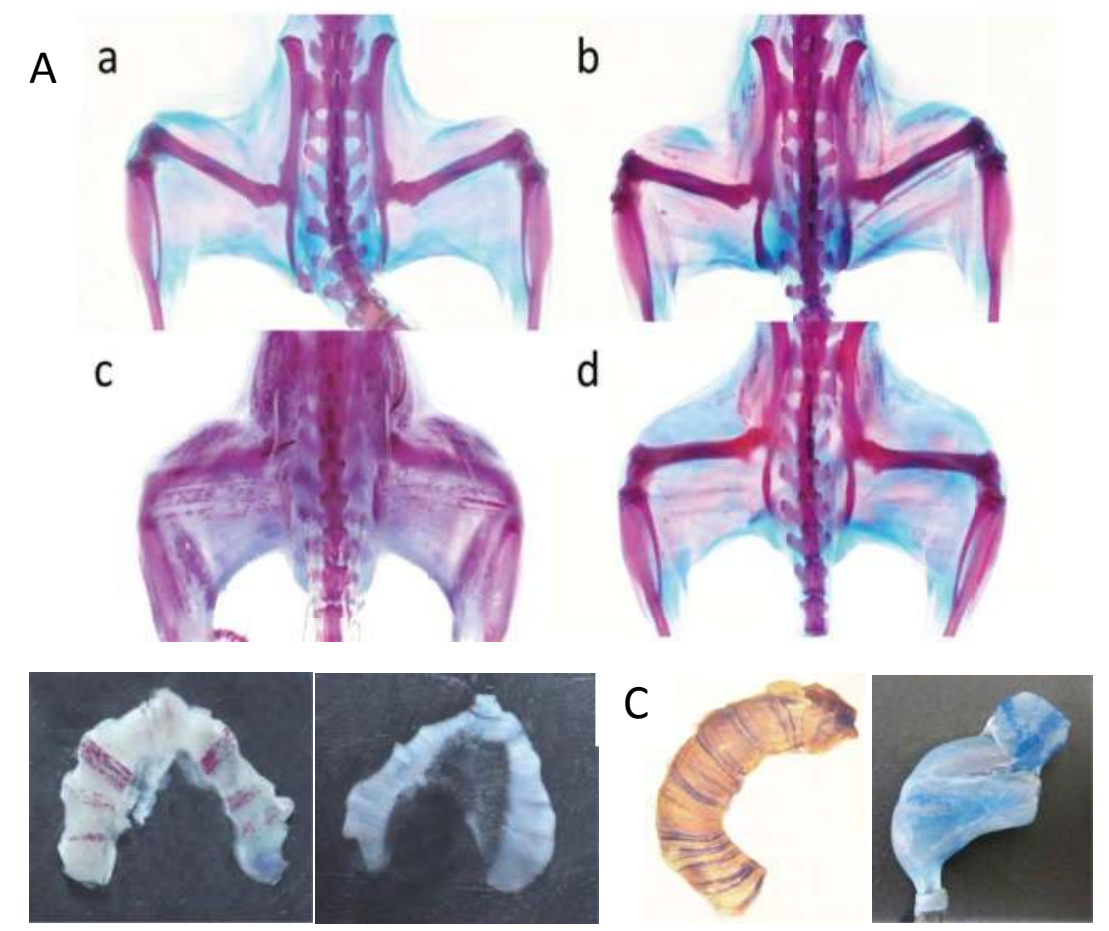

Fig. 4. Results of whole-body double staining of mdx and B10 mice, and Evans blue dye staining of mdx mice. (A) Images of the whole-body double staining of the lower body. (Aa) Lower body of a B10 mouse fed a high-Pi diet. The lower body of mdx mice (A-b) fed a mid-Pi diet, (A-c) high-Pi diet and (A-d) low-Pi diet. (B) Pictures of the whole body double staining of diaphragm. Diaphragm of an mdx mouse fed a high-Pi diet (left) and low-Pi diet (right). (C) Evans blue dye in the diaphragm (left) and lower limb (right) of an mdx mouse. Evans blue-positive lesions are seen in blue.

Quadriceps muscle samples from low-Pi, mid-Pi, and high-Pi fed mdx mice at 30, 60, and 90 days of age were sectioned at $8 \mu \mathrm{m}$ thickness to determine the onset of calcifying lesions. Hematoxylin and eosin (H\&E) and alizarin red $S(1 \%)$ staining were used to observe pathology and detect calcification in the samples (Fig. 5). Histology showed early mineralization in degenerating myofibers in high-Pi fed mdx mice at 30 days of age (only fed a high-Pi diet only for 10 days), whereas no alizarin red-positive areas were present in either mid-Pi or low-Pi fed mdx mice of the same age. In addition, few calcium deposits were seen in mid-Pi fed mdx mice by the age of 60 days or in low-Pi fed mdx mice even by 
90 days of age. Calcium deposits were extensive throughout the entire sections of high-Pi fed mdx mice at 90 days of age.

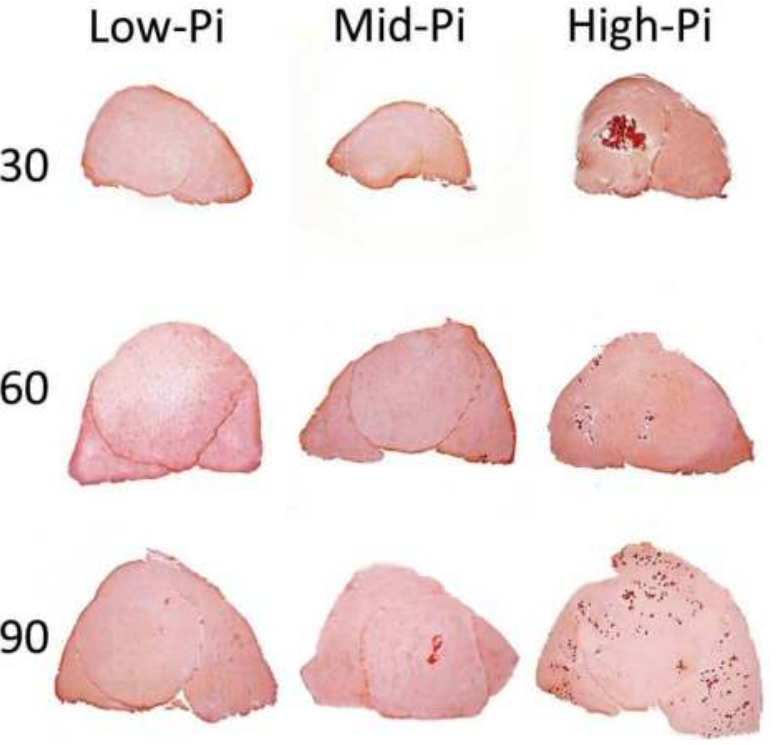

Fig. 5. Alizarin red S-stained cryosections of $\mathrm{mdx}$ mice quadriceps muscle. Calcium deposits are stained red.

\subsection{Ectopic calcification in other tissues}

Although the presence of calcification is rarely reported in organs of mdx mice other than skeletal muscle, including the heart and kidneys, these mice exhibit abnormal cardiac pathology and function (Zhang et al., 2008) and their myocardium is vulnerable (Costas et al., 2010). Rodent models of muscular dystrophies may have potential for sensitivity to myocardial calcification when challenged by mechanical or chemical stressors, because such calcification is commonly observed in the hamster model of muscular dystrophy (Burbach, 1987). For instance, Elsherif et al. (2008) found that dystrophin and $\beta 1$ integrin doubleknockout mice ( $\beta 1 \mathrm{KOmdx}$ ) show exacerbated myocardiopathy and extensive calcification in the heart, particularly under pregnancy-induced stress. Thus we predicted that high-Pi intake would also affect calcification in the myocardium of $\mathrm{mdx}$ mice.

Calcification in the heart was evaluated by $8 \mu \mathrm{m}$ cryosections of samples from the three Pidiet group mice. We found that high-Pi intake induced relatively few cases of myocardial calcification in mdx mice at both 60 and 90 days of age (4 of 30 samples). The form of the crystallization observed in the heart was similar to that of myofiber calcification, although the amount was considerably less (Fig. 6A, C). The incidence of calcification in the heart was absent in mdx fed mid-Pi or low-Pi diets. None of B10 mice fed any of the three types of phosphate diets exhibited myocardial calcification. 
As previously described, klotho mutant mice display a number of age-related diseases, including soft tissue calcification. Morishita et al. (2010) reported that klotho mice fed a normal diet show kidney calcification, whereas mice fed a low-Pi diet have reduced precipitation of calcium in the kidneys. We found that a high-Pi intake results in slight ectopic calcification in kidneys of mdx mice (Fig. 6B, D) whereas mdx mice fed mid-Pi or low-Pi diets showed no evidence of calcium deposition in the kidneys. Similar to the findings in the heart, B10 mice under all phosphate diets also showed no calcification in the kidneys.

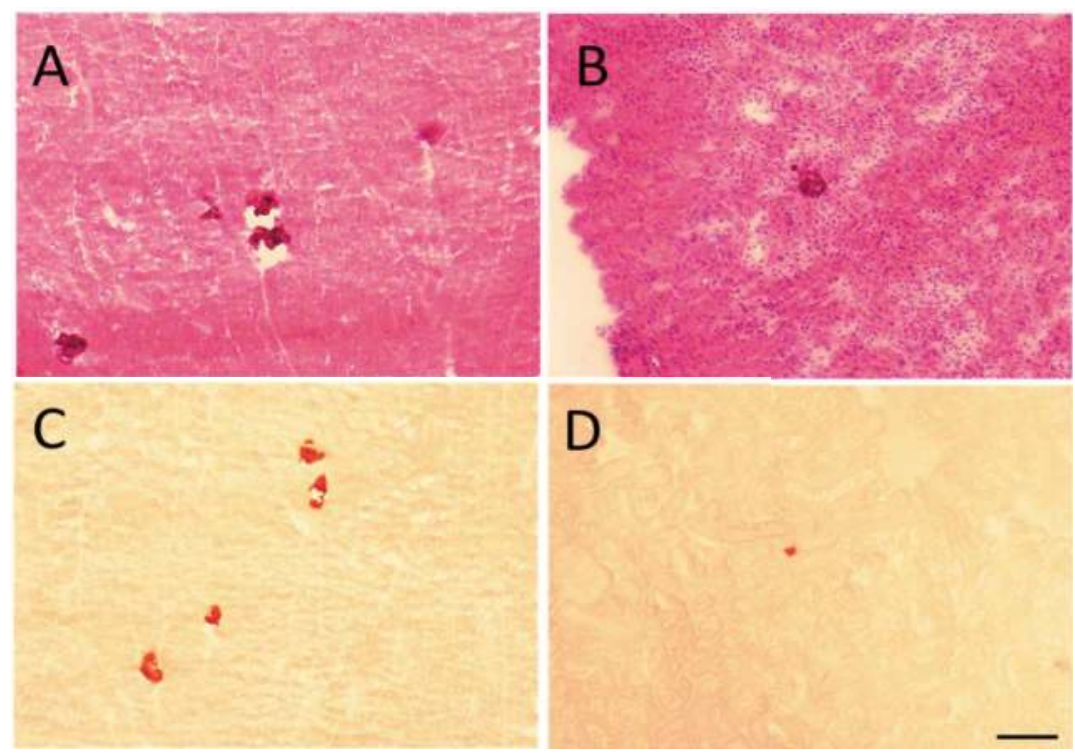

Fig. 6. H\&E and alizarin red S-stained cryosections of the heart and kidneys of an mdx mouse fed a high-Pi diet. H\&E-stained cryosections of the heart (A) and kidney (B). Alizarin red S-stained cryosections of the heart (C) and kidney (D). The bar represents $100 \mu \mathrm{m}$.

\subsection{Changes in serum biochemistry}

We also examined the serum calcium and phosphate concentrations of B10 and mdx mice fed the three types of Pi diets. The serum phosphate levels of high-Pi fed mdx mice were significantly higher than those of B10 mice fed the same diet, and mdx mice under mid-Pi and low-Pi diets (Fig. 7). However, no marked differences in serum calcium concentrations of $\mathrm{mdx}$ mice were detect in the different diet groups. Serum phosphate concentration is largely influenced by dietary intake, with the over-consumption of phosphate often resuting to cause hyperphosphatemia (Calvo et al., 1994), secondary hyperparathyroidism with bone re-sorption (Lutwak et al., 1975), and bone loss (Draper et al., 1979). It is likely that high phosphate intake leads to overworked kidneys and a reduced rate of calcium and phosphate filtration. 


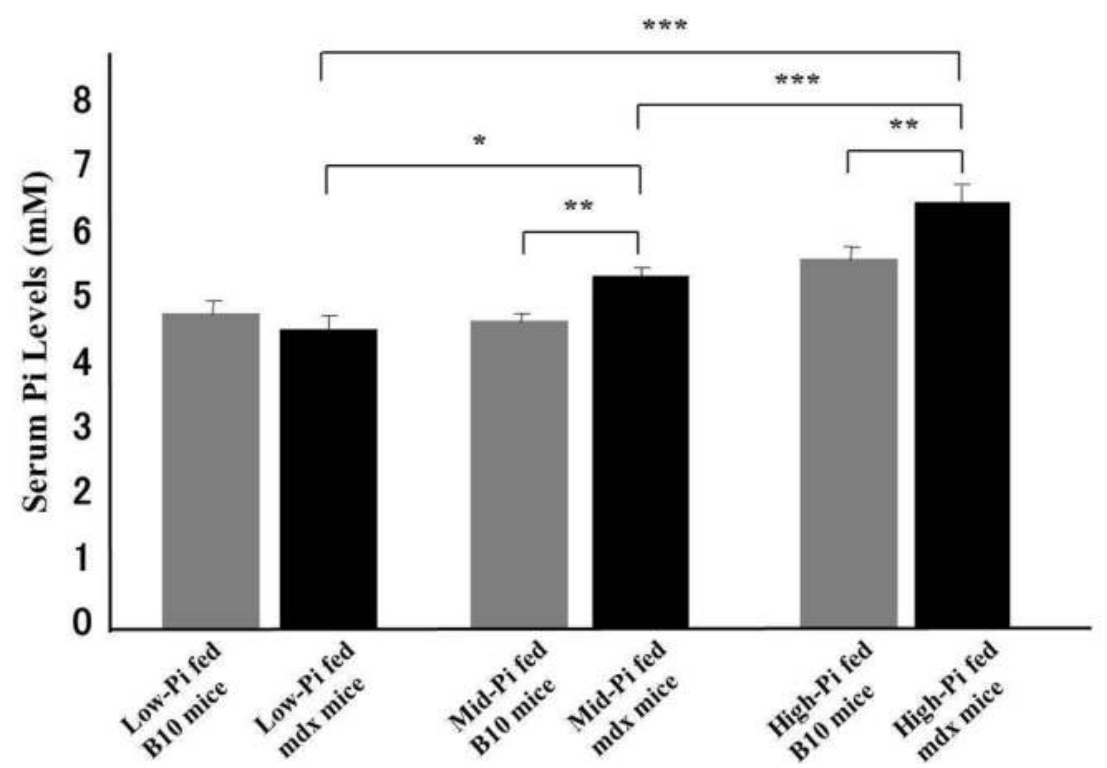

Fig. 7. Serum Pi levels of three-month-old B10 and mdx mice fed three types of Pi diets. Comparison of B10 and mdx mice fed the three Pi diets. $\left({ }^{*}: p<0.05 ;{ }^{* *}: p<0.01 ;{ }^{* * *}: p<0.001\right)$.

\section{Effects of ectopic calcification on muscle function of $\mathrm{mdx}$ mice}

High-Pi intake induced severe ectopic calcification throughout the skeletal muscle of mdx mice. The presence of ectopic calcification in muscles appeared to have a negative impact on the force output of skeletal muscle. To date, no studies have reported the pathophysiological effects of the accumulation of calcium phosphate in muscles. For this reason, we have investigated the effects of ectopic calcification on skeletal muscle contraction of mdx mice.

For muscle force measurements, in situ maximal isometric twitch force and tetanic force of right triceps surae muscle (TSM) were recorded. The isometric force recording system was custom-made and the experimental protocols were based on the design of Dorchies et al. (2006). Sixty-day-old B10 and mdx mice fed the three phosphate diets were lightly anesthetized by diethylether gas and then immobilized on a cork board by covering the bodies with Novix-II (Asahi Techno Glass, Chiba, Japan). A confined area of skin and myofasia of the right hindlimb was cut and exposed, and the sciatic nerve was dissected to induce the analgesic conditions. The knee joint was firmly immobilized by a needle that served as the fulcrum and the Achilles tendon of the leg was then severed and connected to a platinum electrode clip associated with a force transducer (DS2-50N Digital Force Gauge; Imada, Aichi, Japan). A second platinum electrode was directly inserted into the TSM. Experimental trials were started after the animals recovered from anesthesia. Using this procedure, we avoided negative effects (i.e. muscle relaxation) of the anesthetic regimen, which we previously confirmed and were able to collect real data without any disturbances. For the measurement of maximal single twitch, muscles were stimulated with a square wave pulse (0.5-msec duration) of stimulation voltage. Tetanic force was measured with $200-\mathrm{msec}$ bursts of frequency set to $100 \mathrm{~Hz}$. Muscle length and weight of TSM were measured to estimate the cross-sectional area 
(CSA) of the muscle (in $\mathrm{mm}^{2}$ ). The specific twitch and tetanic force were normalized by dividing the measured force by the CSA. Using manual settings of the optimal muscle length, maximal twitch contractions were measured within trials up to 20 contractions and all tetanic force measurements were made at locations where the single twitch force was the greatest.

\subsection{Results of maximal single force (MSF) and maximal tetanic force (MTF) measurements}

Pre-tests results revealed that B10 mice fed the normal CE-2 diet had significantly stronger maximal single force in response to single-pulse stimulation than that of mdx mice (data not shown). This result is consistent with a previous study by Dorchies et al. (2006). Furthermore, although mdx mice have a heavier body weight and muscle mass of the TSM, they exhibited weaker muscle force compared with the control mice. This finding was also consisted with that reported previously (Quinlan et al., 1992), although the muscle mass of anterior tibial muscle was compared, rather than TSM. Therefore, we are confident that our isometric force recording system can be used to evaluate and compare muscle forces between B10 and mdx mice fed the different phosphate diets (Table 1).

We did not detect any significant differences in twitch force between B10 mice of the three phosphate diets groups. However, the high-Pi diet $\mathrm{mdx}$ mice had significantly lower $(p<0.001)$ single force than that of $\mathrm{mdx}$ mice fed a mid-Pi diet (Fig. 8A), while maximal single force was significantly higher in $\mathrm{mdx}$ mice fed a low-Pi diet compared with mid-Pi diet $\mathrm{mdx}$ mice. Notably, however, this value was still lower ( $25 \%$ less $)$ than the corresponding value of B10 mice fed a low-Pi diet.

The maximal tetanic force in response to burst stimulation was also measured for all mice. Similar to the results of twitch force, B10 mice had significantly higher tetanic force than $\mathrm{mdx}$ mice for all three phosphate diets, whereas no marked differences were detected among B10 mice. Mdx mice fed a high-Pi diet produced significantly less $(p<0.001)$ tetanic force than the other mdx mice (Fig. 8B). Based on these findings, we conclude that high-Pi diet has a greater influence on generating the tetanic force in $\mathrm{mdx}$ mice than producing twitch force. These results strongly suggest that calcium deposits in muscles interfere with muscle function. The improvement of muscle forces was likely due to the reduction of ectopic calcification because low Pi-diet did not have a positive effect on force generation in B10 mice, which have no ectopic calcification. However, it is also likely that other factors related to dietary phosphate restriction also contribute to improving muscle function.

\begin{tabular}{|c|c|c|c|c|}
\hline Mouse & $\#$ & Weight $(\mathrm{g})$ & $\mathrm{MSF}\left(\mathrm{mN} / \mathrm{mm}^{2}\right)$ & $\mathrm{MTF}\left(\mathrm{mN} / \mathrm{mm}^{2}\right)$ \\
\hline Low-Pi B10 & 7 & $23.1 \pm 1.0$ & $102.5 \pm 4.6$ & $344.8 \pm 15.2$ \\
\hline Low-Pi mdx & 7 & $23.8 \pm 1.4$ & $74.2 \pm 2.4$ & $254.9 \pm 14.6$ \\
\hline Mid-Pi B10 & 7 & $22.3 \pm 0.7$ & $100.0 \pm 4.1$ & $341.0 \pm 11.1$ \\
\hline Mid-Pi mdx & 7 & $25.2 \pm 0.5$ & $66.8 \pm 1.5$ & $246.4 \pm 7.2$ \\
\hline High-Pi B10 & 7 & $22.8 \pm 0.7$ & $101.0 \pm 3.3$ & $335.6 \pm 6.3$ \\
\hline High-Pi mdx & 7 & $23.6 \pm 0.7$ & $59.6 \pm 2.0$ & $193.9 \pm 9.6$ \\
\hline
\end{tabular}

Table 1. Results of MSF and MTFmeasurements of B10 and mdx mice for the three Pi diet conditions. 

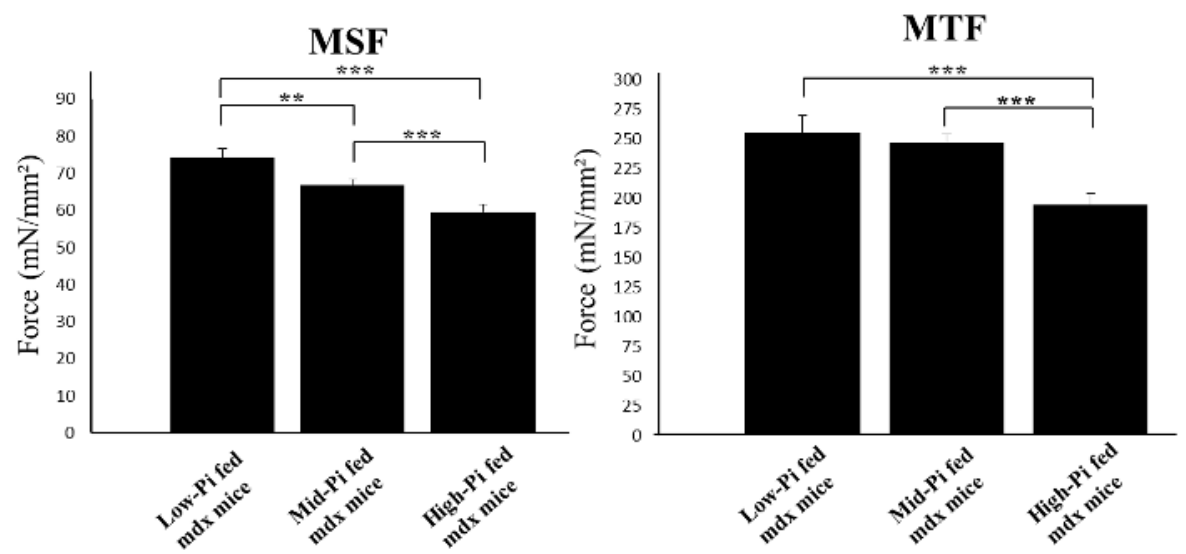

Fig. 8. MSF and MTF measurements of mdx mice for the three Pi diet conditions. (A) Results of MSF. (B) Results of MTF (**: $p<0.01 ;{ }^{* * *}$ p $\left.<0.001\right)$.

\section{Reduced calcification by low Pi diet in a longitudinal study}

Although the influence of dietary phosphate intake on the precipitation of calcium in $\mathrm{mdx}$ mice skeletal muscles has been clarified, the effects of phosphate restriction on severe ectopic calcification remained unclear. To understand the impact of phosphate restriction on the deposition of calcium, a longitudinal study was conducted for four mdx mice raised on highPi diet from weaning to 60 days of age. At age of 60 days, whole-body images of the mdx mice were taken by noninvasive CT scanning using a Latheta LTC-200 X-ray micro CT scanner (Hitachi Aloka Medical, Tokyo, Japan) (Fig. 9). The mdx mice were then divided into two groups, a continuously fed high-Pi diet group and a low-Pi diet group, until the age of 90 days, at which point whole-body images of the mice were taken again. The whole-body images and volume density of ectopic calcification in the lower body (from the top of os coxae to ankle joint) were compared (Fig. 10). Mice fed a high-Pi diet displayed an increased volume (0.066 $\mathrm{cm}^{3}$ ) of ectopic calcification from 60 to 90 days of age, whereas mdx mice fed a low-Pi diet had a reduced $\left(-0.007 \mathrm{~cm}^{3}\right)$. Thus, it was concluded that the restriction the restriction of dietary phosphate from the age of 60 days reduced the pre-formed ectopic calcification within one month, while continuously feeding the mice a high-Pi diet led to more severe calcium deposits.

\section{Mechanisms of calcification}

The complete mechanism underlying progressive muscle degeneration due to dystrophin deficit is unclear. Dystrophin-deficient muscles are highly susceptible to the oxidative stress that results from the early onset of muscle degeneration. Muscle necrosis actively occurs following the degeneration, leading to fibrosis and calcification of muscle fibers (Vercherat et al., 2009). It has been suggested that vascular calcification is actively regulated by osteogenic gene expression in vascular smooth muscle cells (Giachelli, 1999). Attention has been focused on inorganic phosphate as one of the potential factors regulating the observed cellular phenotypic changes, as smooth muscle cells in vitro cultured under high-Pi conditions undergo osteogenesis and form calcium deposits (Jono et al., 2000). As skeletal muscle satellite cells possess multilineage potential (Asakura et al., 2001; Wada et al., 2002), they might also undergo osteogenic differentiation under high-Pi conditions. 
A

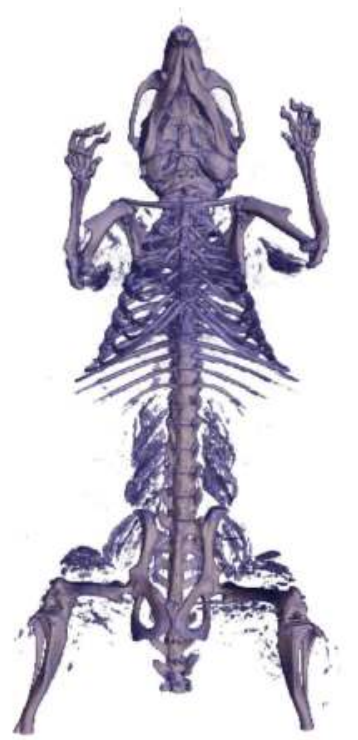

C

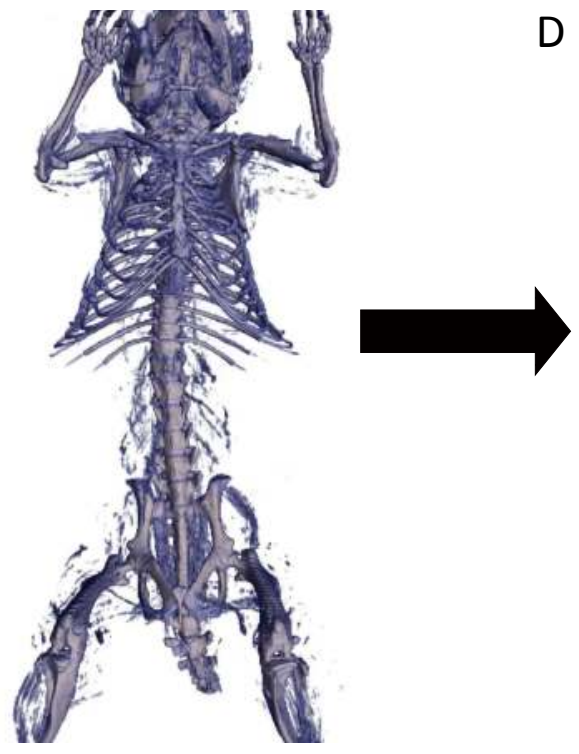

B
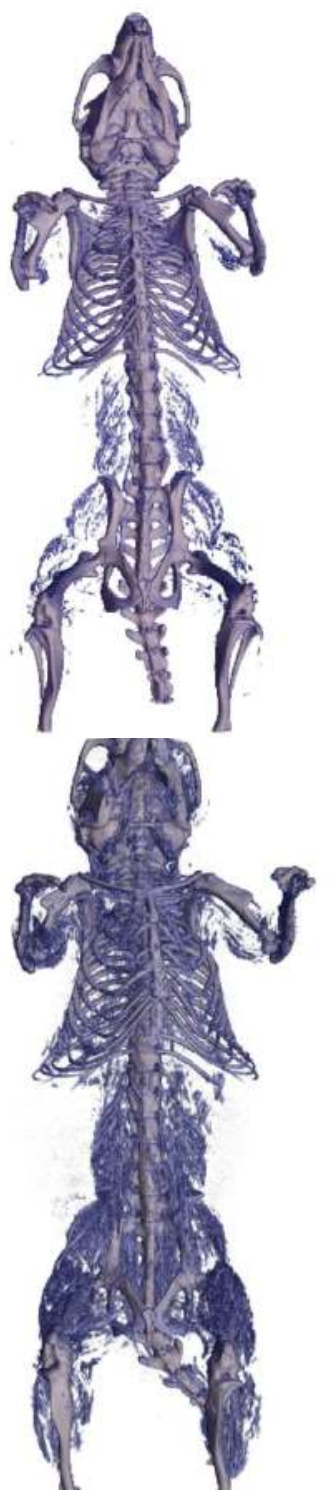

Fig. 9. 3D images of 60-day-old mdx mice fed a high-Pi diet (left) and the images of the same mice after 30 days (A,C) Sixty-day-old mdx mice fed a high-Pi diet. (B) The same mdx mice (90-day-old) fed a low-Pi diet for 30 days. (D) The same mdx mice (90-day-old) fed a high-Pi diet for 30 days. Bones are shown in grey and ectopic calcification is in light blue. 

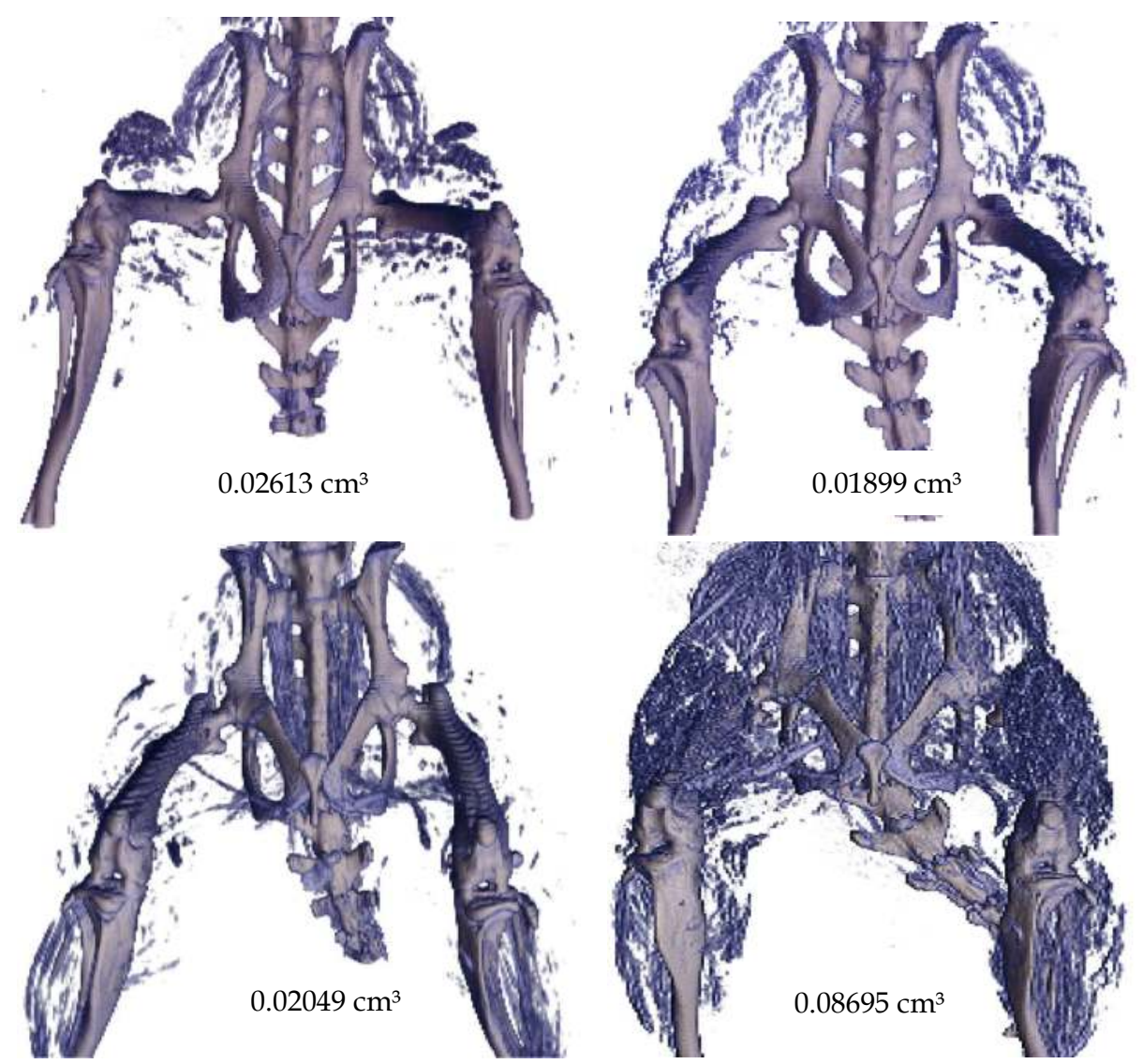

Fig. 10. Enlarged 3D images of the lower limbs of mdx mice fed a high-Pi diet (left) and the images of the same mice fed either low-Pi (top right) or high-Pi diets (bottom right) for 30 days. The numbers represent the volume densities of ectopic calcification in the lower body (from the top of os coxae to ankle joint).

\section{1 $\mathrm{Pi}$-induced osteogenesis and reduced myogenesis in $\mathrm{C} 2 \mathrm{C} 12$ cells}

To study the effects of Pi on muscle cell differentiation, murine myoblast-derived C2C12 cells were cultured for four days under various Pi concentrations and then immunostained for the presence of myogenic (myosin heavy chain; MyHC) and osteogenic (Matrix Gla Protein; MGP) markers. When cultured in normal differentiation medium ( $\mathrm{Pi}=1 \mathrm{mM})$, the cells underwent muscle differentiation and formed myotubes. Myogenesis proceeded until the Pi concentration of the differentiation medium reached $5 \mathrm{mM}$, while myotube formation was strongly suppressed at $7 \mathrm{mM}$. (Fig. 11).

The expression of Runx2, a transcription factor of osteogenesis, increased with the rise of the $\mathrm{Pi}$ concentration (Fig. 12A). The retardation of myogenesis caused by the high $\mathrm{Pi}$ concentration was also evident by the decrease in both the fusion index and myogenin 
expression (Fig. 12A). It was notable that in medium containing $5 \mathrm{mM} \mathrm{Pi}$, myogenesis was not inhibited and the $\mathrm{C} 2 \mathrm{C} 12$ cells differentiated into myotubes, while the expression Runx2 was augmented (Fig. 12B). Further observation revealed that myogenin and Runx2 did not colocalize in the nuclei of myotubes, rather, Runx2 was localized in the cytoplasm. This finding suggests that Runx 2 is inactive in myogenic cells, as it has been reported that Runx2 activity is regulated by translocation between the nucleus and cytoplasm (Zaidi et al., 2001). Upregulation of Runx2 expression was observed by Western blotting not only when the $\mathrm{C} 2 \mathrm{C} 12$ cells were cultured under high-Pi conditions, but also when cultured in the presence of calcium deposits, which were generated by the addition of sodium phosphate and calcium chloride to the medium (Fig. 12C). Osteocalcin, another osteogenic marker which is a secreted protein whose expression is regulated by Runx2, was also examined (Fig 12D). RT-PCR was performed with RNA samples prepared from $\mathrm{C} 2 \mathrm{C} 12$ cells cultured under the various Pi concentrations for four days. Osteocalcin expression was undetectable when the cells were cultured with $1 \mathrm{mM} \mathrm{Pi}$, but increased with the elevation of the Pi concentration. We also measured calcium deposition in $\mathrm{C} 2 \mathrm{C} 12$ cells cultured under the various $\mathrm{Pi}$ concentrations and found that although the cells did not deposit calcium under normal Pi conditions, cells cultured in medium containing $3 \mathrm{mM}$ Pi or higher deposited calcium (alizarin red S-positive cells; Fig. 12E ). The amount of calcium deposits increased significantly at higher Pi concentrations.
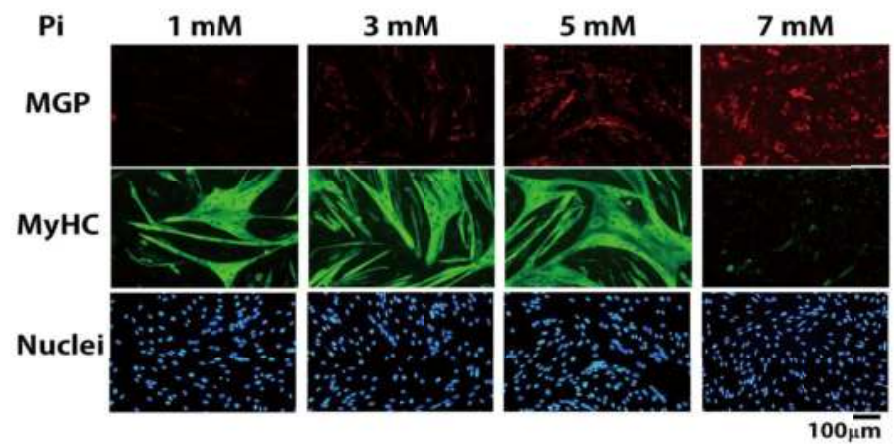

Fig. 11. Immunocytochemistry of C2C12 cells cultured under various Pi concentrations. Cells were immunostained for MyHC (green), MGP (red), and nuclei (blue).

\subsection{Pi-induced calcification in primary cultures of skeletal muscle cells}

Cells isolated from mdx skeletal muscle tissue were cultured in normal $\mathrm{Pi}(1.3 \mathrm{mM})$ to high$\mathrm{Pi}(5 \mathrm{mM})$ medium to study the effects of Pi in primary culture cells. The cells formed myotubes when cultured in normal medium, whereas myotube formation was strongly inhibited under high-Pi conditions. The results of both alizarin red S and von Kossa staining revealed that numerous calcium deposits were present in cells after ten days of culture in high-Pi medium, but none detected in cells cultured in normal medium (Fig. 13). Therefore, $\mathrm{Pi}$ induces osteogenesis in myoblasts, resulting in calcification while inhibiting myogenesis. We conclude that the calcification of skeletal muscle is mainly due to the elevation of intracellular Pi levels. 

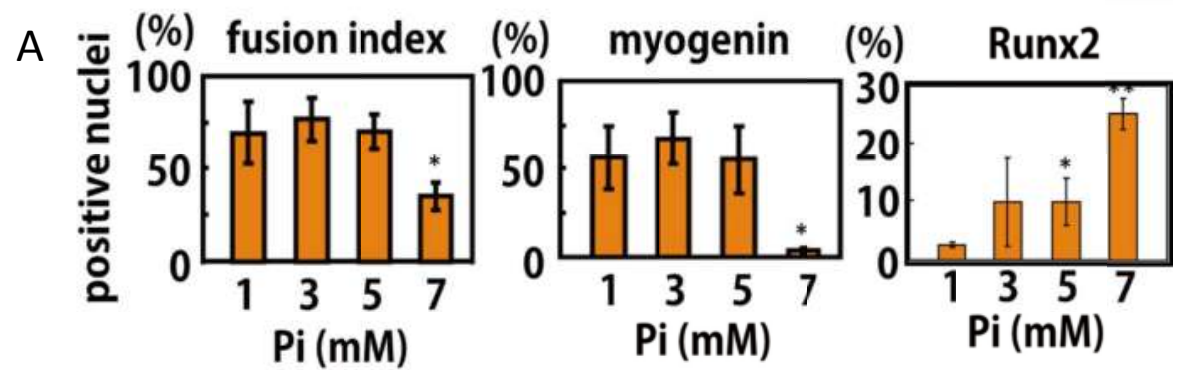

B

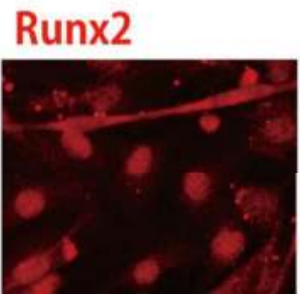

myogenin

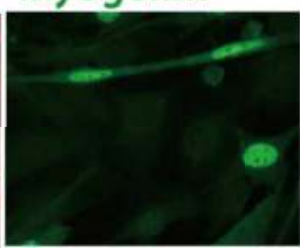

merge

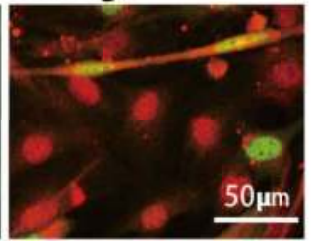

C
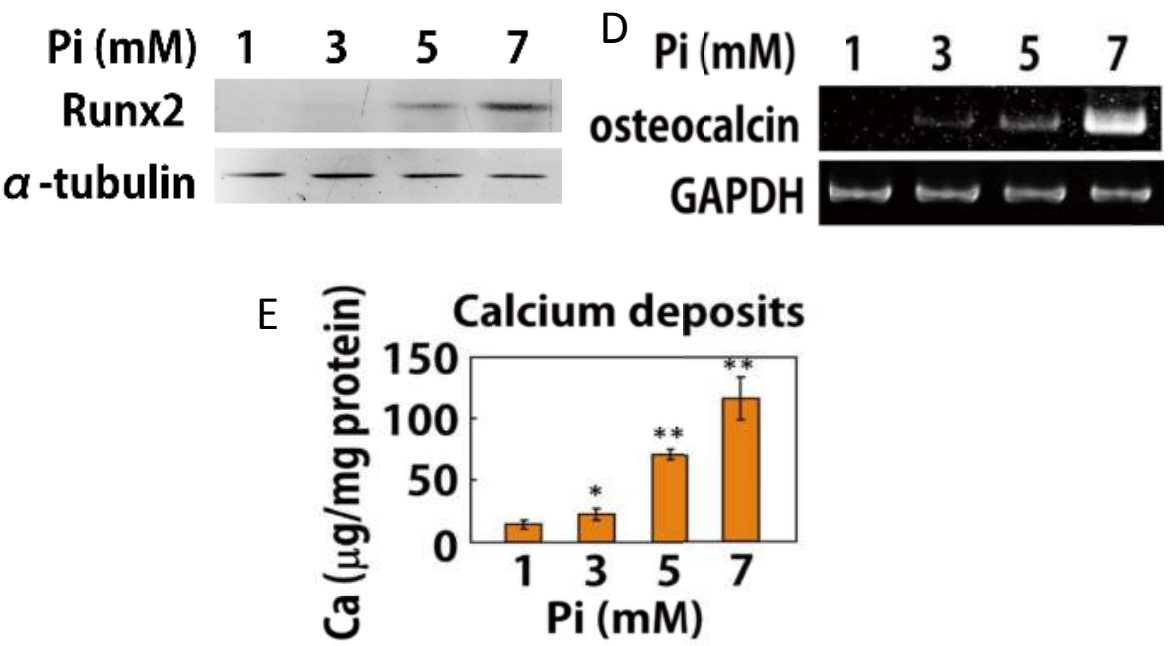

Fig. 12. Immunocytochemistry and RT-PCR of C2C12 cells cultured under various Pi concentrations $(1,3,5$, and $7 \mathrm{~m})$. (A) The fusion index, myogenin expression, and expression of Runx 2 were quantified. The fusion index and ratio of nuclei expressing myogenin decreased, while the ratio of Runx2-expressing nuclei increased with increasing Pi concentration. (B) Close observation of cells cultured in medium containing $5 \mathrm{mM}$ Pi by staining with Hoechst 33258 to show the nuclei, or immunostained for myogenin or Runx2.

(C) Western blotting of C2C12 cells cultured under increased Pi or Ca concentrations. (D) RT-PCR for osteocalcin in C2C12 cells cultured under various Pi concentrations. (E) Quantification of calcium deposites generated by $\mathrm{C} 2 \mathrm{C} 12$ cells cultured under various $\mathrm{Pi}$ concentrations. $\left(*: p<0.05 ;{ }^{* *}: \mathrm{p}<0.01\right)$. 


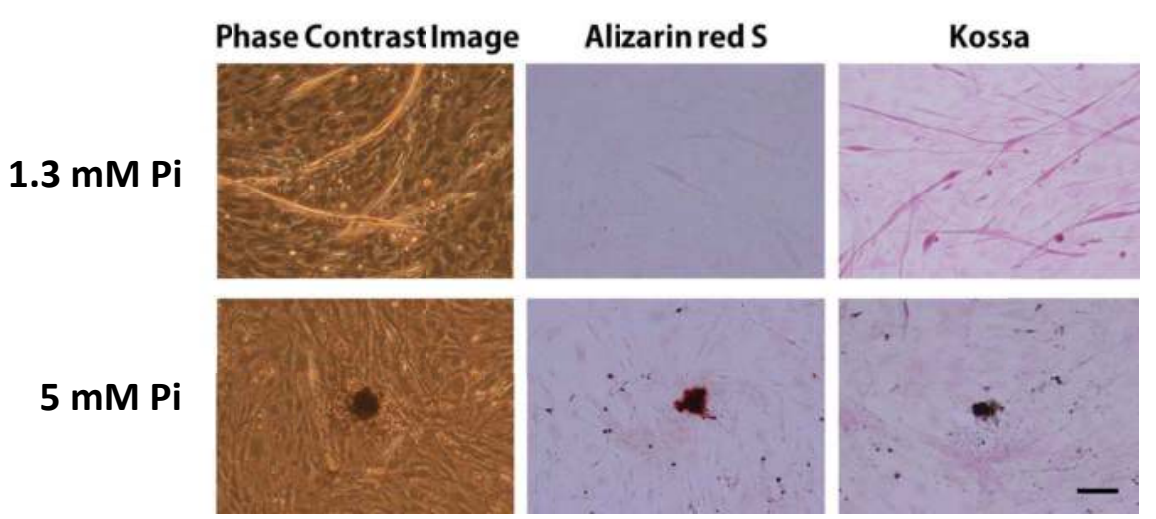

Fig. 13. Calcification of mdx mouse muscle-derived primary culture cells. Calcium deposits were stained red or black with alizarin red S and von Kossa staining, respectively. No calcification was observed when cells were cultured in normal medium containing $1.3 \mathrm{mM}$ Pi. The von Kossa-stained samples were counterstained with nuclear fast red, and myotubes appear pink. The bar represents $100 \mu \mathrm{m}$.

\section{Conclusion}

In this study, we reviewed the mechanisms underlying calcification in skeletal muscle cells following the elevation of intracellular Pi concentrations and revealed the effects of dietary phosphate intake on ectopic calcification in $\mathrm{mdx}$ mice. Both in vivo and in vitro, high-Pi conditions lead to the precipitation of calcium in $\mathrm{mdx}$ mice. We have demonstrated that the presence of ectopic calcification in skeletal muscle exacerbates the impaired muscle function of mdx mice, which represents a novel finding. The main goal of our studies is to understand the effects and efficacy of nutritional components on muscular dystrophy as a prior therapy. The effects of dietary phosphate intake on muscle pathology and kidney function need to further elucidated in future studies. Furthermore, the therapeutic potential of nutrition, particularly phosphate intake, should be considered when treating patients with DMD.

\section{Acknowledgement}

This work has been supported by Health and Labour Sciences Research Grant (19A-020) for Research on Psychiatric and Neurological Diseases and Mental Health, Intramural Research Grant(23-5) for Neurological and Psychiatric Disorders of NCNP and a Research Grant for Nervous and Mental Disorders [20B-13] from the Ministry of Health, Labour and Welfare, Japan.

\section{References}

Asakura, A.; Komaki, M. \& Rudnicki, M. (2001). Muscle Satellite Cells are Multipotential Stem Cells that Exhibit Myogenic, Osteogenic, and Adipogenic Differentiation. Differentiation, Vol.68, pp.245-253. 
Burbach, J. (1987). Ultrastructure of Cardiocyte Degeneration and Myocardial Calcification in the Dystrophic Hamster. The American Journal of Anatomy, Vol.179, pp.291-307.

Calvo, M. (1994). The Effects of High Phosphoru Intake on Calcium Homeostasis. Advances in Nutritional Researches, Vol.9, pp.183-207.

Costas, J.; Nye, D.; Henley, J. \& Plochocki, J. (2010). Voluntary Exercise Induces Structural Remodeling in the Hearts of Dystrophin-deficient Mice. Muscle Nerve, Vol.42, pp.881-885.

Coulton, G.; Morgan, J.; Partridge, T. \& Sloper, J. (1988). The mdx Mouse Skeletal Muscle Myopathy: I. A Histological, Morphometric and Biochemical Investigation. Neuropathology and Applies Neurobiology, Vol.14, pp.53-70.

Dingerkus, G. \& Uhler, L. (1977). Enzyme Clearing of Alcian Blue Stained Whole Small Vertebrates for Demonstration of Cartilage. Biotechnic \& Histochemistry, Vol.52, pp.229-232.

Dorchies, O.; Wagner, S.; Vuadens, O.; Waldhauser, K.; Buetler, T.; Kucera, P. \& Ruegg, U. (2006). Green Tea Extract and Its Major Polyphenol(-)-epigallocatechin Gallate Improve Muscle Function in a Mouse Model of Duchenne Muscular Dystrophy. American Journal of Physiology. Cell Physiology, Vol.290, pp.616-625.

Draper, H. \& Bell, R. (1979). Nutrition and Osteoporosis. Advances in Nutritional Researches, Vol.145, pp.389-391.

El-Abbadi, M.; Pai, A.; Leaf, E.; Yang, H.; Bartley, B.; Quan, K.; Ingalls, C.; Liao, H. \& Giachelli, C. (2009). Phosphate Feeding Induces Arterial Medial Calcification in Uremic Mice: Role of Serum Phosphorus, Fibroblast Growth Factor-23, and Osteopontin. Kidney International, Vol.75, pp.1297-1307.

Elsherif, L.; Huang, M.; Shai, S.; Yang, Y.; Li, R.; Chun, J.; Mekany, M.; Chu, A.; Kaufman, S. \& Ross, R. (2008). Combined Deficiency of Dystrophin and $\beta 1$ Integrin in Cardiac Myocyte Causes Myocardial Dysfunction, Fibrosis and Calcification. Circulation Research, Vol.102, pp.1109-1117.

Gaschen, F.; Hoffman, E.; Gorospe, J.; Uhl, E.; Senior, D.; Cardinet, G. \& Pearce, L. (1992). Dystrophin Deficiency Causes Lethal Muscle Hypertrophy in Cats. Journal of the Neurological Sciences, Vol.110, pp.149-159.

Giachelli, C. (2009). The Emerging Role of Phosphate in Vascular Calcification. Kidney International, V0175, pp.890-897.

$\mathrm{Hu}$, M.; Kuro-o,M. \& Moe, O. (2010). Klotho and Kidney Disease. Journal of Nephrology, Vol.16, pp.136-144.

Kendrick, J.; Kestenbaum, B. \& Chonchol, M. (2011). Phosphate and Cardiovascular Disease. Advances in Chronic Kidney Disease, Vol.18, pp.113-119.

Khan, M. (1993). Corticosteroid Therapy in Duchenne Muscular Dystrophy. Journal of the Neurological Sciences, V01.120, pp.8-14.

Kikkawa, M.; Ohno, T.; Nagata, Y.; Shiozuka, M.; Kogure, T. \& Matsuda, R. (2009). Ectopic Calcification is Caused by Elevated Levels of Serum Inorganic Phosphate in $\mathrm{Mdx}$ Mice. Cell Structure and Function, Vol.34, pp.77-88. 
Korff, S.; Riechert, N.; Schoensiegel, F.; Weichenhan, D.; Autschbach, F.; Katus, H. \& Ivandic, B. (2006). Calcification of Myocardial Necrosis is Common in Mice. Virchows Archiv, Vol.448, pp.630-638.

Kuro-o. (2010). Overview of the FGF-23-Klotho Axis. Pediatric Nephrology, Vol.25, pp.583590.

Kuro-o, M.; Matsumura, Y.; Aizawa, H.; Kawaguchi, H.; Suga, T.; Utsugi, T.; Ohyama, Y.; Kurabayashi, M.; Kaname, T.; Kume, E.; Iwasaki, H.; Iida, A.; Shiraki-Iida, T.; Nishikawa, S.; Nagai, R. \& Nabeshima, Y. (1997). Mutation of the Mouse Klotho Gene Leads to a Syndrome Resembling Ageing. Nature, Vol.309, pp.4551.

Liu, J.; Okamura, C.; Bogan, D.; Bogan, J.; Childers, M. \& Kornegay. J. (2004). Effects of Prednisone in Canine Muscular Dystrophy. Muscle Nerve, Vol.30, pp.767-773.

Lutwak, L. (1975). Metabolic and Biochemical Considerations of Bone. Annals of Clinical and Laboratory Science, Vol.5, pp.185-194.

Matsuda, R.; Nishikawa, A. \& Tanaka, H. (1995). Visualization of Dystrophic Muscle Fibers in $\mathrm{Mdx}$ Mouse by Vital Staining with Evans Blue: Evidence of Apoptosis in Dystrophin-Deficient Muscle. Journal of Biochemistry, Vol.118, pp.959-964.

McLeod, M. (1980). Differential Staining of Cartilage and Bone in Whole Mouse Fetuses by Alcian Blue and Alizarin Red S. Teratology, Vol.22, pp.229-301.

Morishita, K.; Shirai, A.; Kubota, M.; Katakura, Y.; Nabeshima, Y.; Takeshige, K. \& Kamiya, T. (2010). The Progression of Aging in Klotho Mutant Mice Can Be Modified by Dietary Phosphorus and Zinc. The Journal of Nutrition, Vol.131, pp.3182-3188.

Nguyen, F.; Cherel, L.; Guigand, I.; Goubault-Leroux \& Myers, M. (2002). Muscle Lesions Associated with Dystrophin Deficiency in Neonatal Golden Retriver Puppies. Journal of Comparative Pathology, Vol.126, pp.100-108.

Quinlan, J.; Johnson, S.; McKee, M. \& Lyden, S. (1992). Twitch and Tetanus in mdx Mouse Muscle. Muscle Nerve, Vol.15, pp.837-842.

Razzaque, M.; Sitara, D.; Taguchi, T.; St-Arnaud, R. \& Lanske, B. (2006). Premature Aginglike Phenotype in Fibroblast Growth Factor 23 Null Mice is a Vitamin D Mediated Process. The FASEB Journal, Vol.20, pp.720-722.

Vercherat, C.; Chung, T.; Yalcin, S.; Gulbagci, N.; Gopinadhan, S.; Ghaffari, S. \& Taneja, R. (2009). Stra13 Regulates Oxidative Stress Mediated Skeletal Muscle Degeneration. Human Molecular Genetics, Vol.18, pp.4304-4316.

Verma, M.; Asakura, Y.; Hirai, H.; Watanabe, S.; Tastad, C.; Fong, G.; Ema, M.; Call, J.; Lowe, D. \& Asakura, A. (2010). Fit-1 Haploinsufficiency Ameliorates Muscular Dystrophy Phenotype by Developmentally Increased Vasculature in Mdx Mice. Human Molecular Genetics, Vol.19, pp.4145-4159.

Wada, M.; Inagawa-Ogashiwa, M.; Shimizu, S.; Yasumoto, S. \& Hashimoto, N. (2002). Generation of Different Fates from Multipotent Muscle Stem Cells. Development, Vol.129, pp.2987-2995.

Webb, G. \& Byrd, R. (1994). Simultaneous Differential Staining of Cartilage and Bone in Rodent Fetuses: an Alcian Blue and Alizarin Red S Procedure Without Glacial Acetic Acid. Biotechnic and Histochemistry, Vol.64, pp.181-185. 
Wong, B. \& Christopher, C. (2002). Corticosteroids in Duchenne Muscular Dystrophy: a Reappraisal. Journal of Child Neurology, Vol.17, pp.183-190.

Zaidi, S.; Javed, A.; Choi, J.; van Wijnen, A.; Stein, J.; Lian, J. \& Stein, G. (2001). A Specific Targeting Signal Directs Runx2/Cbfa1 to Subnuclear Domains and Contributes to Transactivation of the Osteocalcin Gene. Journal of Cell Sciences, Vol.114, pp.30933102.

Zhang, W.; Hove, M.; Schneider, J.; Stuckey, D.; Sebag-Montefiore, L.; Bia, B.; Radda, G.; Davis, K.; Neubauer, S. \& Clarke, K. (2008). Abnormal Cardiac Morphology, Function and Energy Metabolism in the Dystrophic mdx Mouse: An MRI and MRS Study. Journal of Molecular and Cellular Cardiology, Vol.45, pp.754-760. 


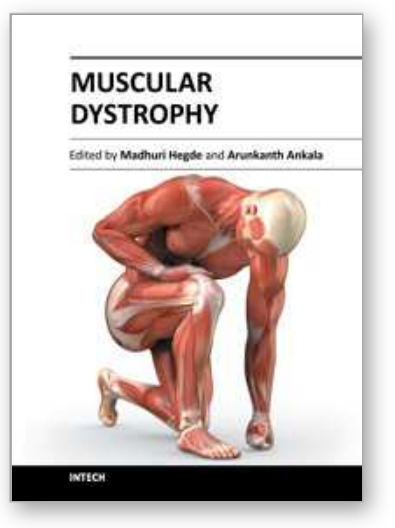

\author{
Muscular Dystrophy \\ Edited by Dr. Madhuri Hegde
}

ISBN 978-953-51-0603-6

Hard cover, 544 pages

Publisher InTech

Published online 09, May, 2012

Published in print edition May, 2012

With more than 30 different types and subtypes known and many more yet to be classified and characterized, muscular dystrophy is a highly heterogeneous group of inherited neuromuscular disorders. This book provides a comprehensive overview of the various types of muscular dystrophies, genes associated with each subtype, disease diagnosis, management as well as available treatment options. Though each different type and subtype of muscular dystrophy is associated with a different causative gene, the majority of them have overlapping clinical presentations, making molecular diagnosis inevitable for both disease diagnosis as well as patient management. This book discusses the currently available diagnostic approaches that have revolutionized clinical research. Pathophysiology of the different muscular dystrophies, multifaceted functions of the involved genes as well as efforts towards diagnosis and effective patient management, are also discussed. Adding value to the book are the included reports on ongoing studies that show a promise for future therapeutic strategies.

\title{
How to reference
}

In order to correctly reference this scholarly work, feel free to copy and paste the following:

Eiji Wada, Namiko Kikkawa, Mizuko Yoshida, Munehiro Date, Tetsuo Higashi and Ryoichi Matsuda (2012). Effects of Dietary Phosphate on Ectopic Calcification and Muscle Function in mdx Mice, Muscular Dystrophy, Dr. Madhuri Hegde (Ed.), ISBN: 978-953-51-0603-6, InTech, Available from:

http://www.intechopen.com/books/muscular-dystrophy/membrane-disruption-of-dystrophic-muscle

\section{INTECH}

open science | open minds

\section{InTech Europe}

University Campus STeP Ri

Slavka Krautzeka 83/A

51000 Rijeka, Croatia

Phone: +385 (51) 770447

Fax: +385 (51) 686166

www.intechopen.com

\section{InTech China}

Unit 405, Office Block, Hotel Equatorial Shanghai

No.65, Yan An Road (West), Shanghai, 200040, China

中国上海市延安西路65号上海国际贵都大饭店办公楼 405 单元

Phone: +86-21-62489820

Fax: $+86-21-62489821$ 
(C) 2012 The Author(s). Licensee IntechOpen. This is an open access article distributed under the terms of the Creative Commons Attribution 3.0 License, which permits unrestricted use, distribution, and reproduction in any medium, provided the original work is properly cited. 